\title{
Medium to high-frequency static DGPS error reduction using multi-resolution de-noising vs. de-trending procedures
}

\author{
Research Article
}

A. A. El-Ghazouly ${ }^{1 *}$, M. M. Elhabiby ${ }^{2}$ and N. M. El-Sheimy ${ }^{1}$

1 Mobile Multi-Sensor Systems (MMSS) Research Group, Department of Geomatics Engineering, the University of Calgary Calgary, Alberta, T2N 1N4, Canada

2 Public Works Department Faculty of Engineering Ain Shams University, Khalifa El-Maamon st, Abbasiya sq., Post Code: 11566, Cairo. Egypt

\begin{abstract}
:
Global positioning systems is known to create bias effects such as multipath, ionospheric and tropospheric delays that behave like lowfrequency noise. Random measurement errors can also occur and these are typically characterized as high-frequency noise. The lowfrequency nature of a multipath is what causes the largest error, which in carrier phase measurements can reach up to $5 \mathrm{~cm}$. For a static base station it is required that both error components (low and high-frequency) are removed and not included in the static baseline processing.

This paper will introduce two different multi-resolution techniques that can be used separately or combined to remove the low to highfrequency GPS errors. The first technique is applied using the wavelets as a de-noising tool to tackle the high-frequency errors in the double difference domain. A detailed analysis is also made to choose the best wavelet base function and threshold technique estimator by comparing different wavelet parameters along with different thresholding techniques. The second technique discussed in this paper uses the wavelets technique as a de-trending tool to tackle the low-frequency portion of the double differenced measurements.

The results of this research paper indicate that the de-trending technique can reduce the double difference errors dramatically for short baselines when compared to the de-noising technique. Conversely, the de-trending technique can cause a biased solution for long baselines, as it will enhance the RMS value and indicate good statistics for the solution. However, the will be shifted from it depending on the low frequency part of the error (ionosphere, low multipath). Therefore, it is important to isolate ionospheric error by modeling (and not spectrum filtering) before dealing with multipath, as it is hard to separate between both errors in the spectral domain.
\end{abstract}

Keywords:

DGPS $\cdot$ de-noising $\cdot$ de-trending $\cdot$ multipath $\bullet$ wavelet

(c) Versita sp. z o.o.

Received 06-06-2013; accepted 28-08-2013

1. Introduction

Multipath mitigation in the final measurements domain (carrier/code observables) is a result of the large amount of residuals from mitigation in the antenna/receiver domain. (Raquet and Lachapelle 1996) used a multi-antenna array to mitigate the multipath error at the GPS reference station. (Han and Rizos 1997) were

\footnotetext{
*E-mail: aaelghaz@ucalgary.ca
}

VERSITA the first to propose the use of finite impulse response (FIR) filters to extract or eliminate multipath. However, this technique has certain limitations because signals (i.e.: crustal deformation) that fall into the same frequency band will be removed.

A more effective technique, based on the use of an adaptive filter to extract and eliminate multipath, was suggested by (Linlin et al. 2000 , Lee 2008). Since GPS observation noise tends to change with time, it was determined to be more appropriate to use an adaptive filter rather than a fixed filter for the purpose of multipath mitigation. The implementation of such a technique is dependent on the selection of an appropriate value for the step-size parameter and 
the filter length. (Zhang and Bartone, 2004) developed a multipath mitigation technique based on the multipath frequency spectrum analysis. They used code minus carrier to model multipath errors and identify window size before the error was transformed into the frequency domain using the Fast Fourier Transform (FFT). In the Fourier coefficients domain, the authors mitigated the multipath error based on the estimated multipath frequency. The reconstruction stage uses the Inverse Fast Fourier Transform (IFFT) to compute the multipath error-reduced code-phase measurements. This technique effectively reduces code multipath error, particularly in the static mode, where the multipath fading frequency is well predicted and the fading frequency ranges from zero to $1 \mathrm{~Hz}$. However, more investigation is required to apply this correction in kinematic mode given the rapid change in multipath frequency.

Wavelets are used extensively as an alternative to FFT analysis because their elements are essentially waveforms indexed by three parameters: position, scale and frequency. This is what produces such strong localized time-frequency properties, which gives the wavelets the ability to provide an accurate location of the transient component in the signal while retaining information about the fundamental frequency. Therefore, wavelet transforms offer advantages over the frequency domain analysis (Fourier analysis) and the time domain analysis (Kalman filter). Most of the research conducted on wavelet multipath mitigation uses wavelet transform on its own or combined with other techniques to mitigate high-frequency multipath error. (Dammalage et al. 2010) used biorthogonal wavelets to de-noise code measurements for DGPS applications and reached a 60\% error reduction. (Ogaja and Satirapod 2007) applied the Symlet base function at the fourth scale decomposition level to detect and separate high-frequency multipath errors from receiver noise when using high-rate $(1-\mathrm{Hz}) \mathrm{GPS}$ data. (Souza and Monico, 2004) investigated the use of both Symlet and Daubechies base functions to reduce the high-frequency multipath in GPS relative positioning. They tested both the hard and soft threshold along with the median threshold estimator and concluded that Symlet 12 along with the hard threshold performed the best and achieved a 30\% error reduction. (Satirapod and Rizos 2005) used wavelets to mitigate multipath at permanent stations. The use of wavelets as a de-noising tool for processing and mitigating multipath error proved to be an effective tool for high-frequency multipath mitigation. In contrast, denoising techniques cannot remove this type of error in medium to low-frequency multipath components. As a result, wavelets should be used differently according the type of errors being mitigated, for high frequency errors wavelet de-noising should be used to mitigate that error while in low frequency error wavelet de-trending should be the method of mitigation. Many of the techniques discussed above already used wavelets as a de-noising tool (Dammalage et al. 2010, Ogaja and Satirapod 2007, Souza and Monico 2004 and Satirapod and Rizos 2005) but it is still not clear which wavelet parameters should be used with GPS double difference data to mitigate the medium to high-frequency errors (mainly multipath and uncorrelated ionospheric error). Moreover, there is no compartative analysis made using different wavelet thresholding estimators or techniques to mitigate the medium to highfrequency errors or the best wavelets denoising technique for GPS error mitigation.

This research paper will introduce two different multi-resolution techniques that can be used separately or combined to remove the low to high-frequency GPS errors. The first technique is applied using the wavelets as a de-noising tool to tackle the highfrequency errors in the double difference domain and to obtain a de-noised double difference signal that can be used in a positioning calculation. A detailed analysis is also made to choose the best wavelet base function and threshold technique estimator by comparing different wavelet parameters along with different thresholding techniques. The second technique discussed in this paper uses the wavelets technique as a de-trending tool to tackle the lowfrequency portion of the double differenced measurements. The de-trended and the de-noised double differenced measurements will be used to compute accurate positions for the baselines length from a few hundred meters to $50 \mathrm{~km}$.

\section{Wavelets De-Noising}

Double difference errors may have low (coarse-gain) and/or high frequency (fine-gain) fluctuations. Fortunately, the highfrequency aspect is relatively easy to remove if the proper denoising threshold is applied. Multi-resolution analysis has been proven to be an important tool for eliminating noise in signals. The strong localization properties of the wavelets in the time and frequency domain allow the wavelets to detect fine and coarse variations in the signal (Hubbard 1998). A basic wavelet de-noising algorithm consists of three steps:

1. Decompose the noisy signal (double difference GPS signal) using a wavelets multi-resolution analysis of its details and approximations.

2. De-noise the details' wavelets coefficients, which contain the high-frequency portion of the signal.

3. Reconstruct the de-noised signal by applying the inverse wavelet transform to de-noised coefficients.

$c^{m}{ }_{n}=\left\langle S, \Phi_{m, n}\right\rangle=\sum_{l \in Z} h_{l}\left\langle S, \Phi_{m-1,2 n+1}\right\rangle=\sum_{l \in Z} h_{l-2 n} c_{l}^{(m-1)}$

$d^{m}{ }_{n}=\left\langle S, \psi_{m, n}\right\rangle=\sum_{l \in Z} g_{l}\left\langle S, \psi_{m-1,2 n+1}\right\rangle=\sum_{l \in Z} g_{l-2 n} c_{l}^{(m-1)}$

The double differenced signal $(S$ ) can be represented by approximation coefficients $c_{n}{ }^{m}$ and $d_{n}{ }^{m}$ detailed part (see Eq. (1) and

VERSITA 
Eq. (2)). The double difference GPS signal is decomposed into two sets of coefficients: low frequency (approximation $d_{n}{ }^{m}$ ) and higher frequency (details $c_{n}{ }^{m}$ ) by convolving the input signal with low pass $(\mathrm{L})$ and high pass $(\mathrm{H})$ filters, $p s i_{m, n}$ is the wavelets function generated from the original mother wavelets $\psi \in L^{2}(\mathrm{R}), \Phi_{m, n}$ is the scaling function, $m$ is the scale or level of decomposition, and $n$ is the shifting or translation integer respectively. One of the main advantages of wavelets is the presence of various parameters that can be controlled to help in the classification and separation of different types of signals with different frequencies. The concept of wavelet analysis is based on finding the similarities between the candidate base function and the signal, therefore the wavelet parameters must be selected to match the properties of the GPS double difference error. Four different parameters are used in this research to create several combinations to detect the optimum combination in reducing the high-frequency GPS errors. These parameters are:

1. Wavelet base function and vanishing moment

2. Level of decomposition

3. Threshold type

4. Threshold estimator

All the possible combinations among the parameters are investigated to ensure that the use of the wavelet transform technique is efficient for GPS error mitigation. These parameters will be described in the next subsections.

\subsection{Wavelet base function and Vanishing moment}

The main criterion for selecting the wavelet base function is that the base function matches the shape of the main error, which in this case is the multipath. Ray (2000) demonstrated the pattern of the carrier phase multipath error in GPS signals, which can take one of two shapes (Figure 1). The multipath error with a small magnitude causes a sinusoidal pattern, while the multipath error with a high magnitude causes a saw-tooth pattern. Therefore, for the purposes of this investigation, the wavelet base function that can match both sinusoidal and saw-tooth pattern will be used. There are a number of wavelet base functions (including Haar, Daubechies, Coiflets, Symlet, Biorthogonal) that differ in the way their scaling and wavelet functions are defined. Wavelets are classified into a family by the number of vanishing moments, $N$. This number, which is weakly linked to the number of oscillations (the more vanishing moments a wavelet has, the more it oscillates), determines what a wavelet does not extract, that is, what it recognizes. Wavelets with one vanishing moment do not recognize a linear function. Therefore, two vanishing moments make wavelets blind to quadratic functions as well as three vanishing moments to cubic functions, and so on. Wavelets with many vanishing moments also yield small coefficients when used to analyze a low frequency (Hubbard 1998). Within each family of wavelets

\section{VERSITA}

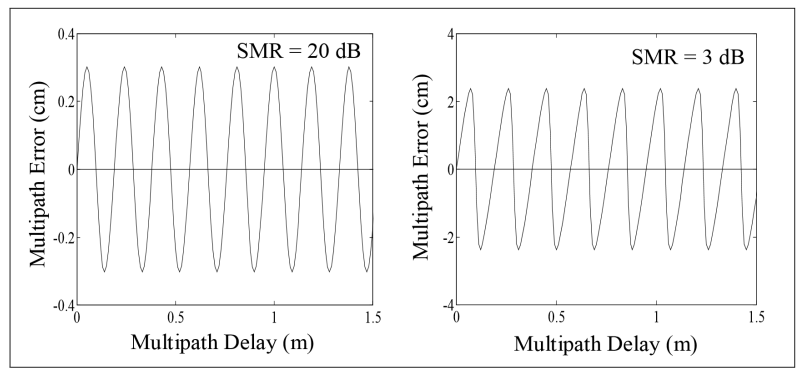

Figure 1. Carrier phase multipath error pattern for short multipath delays due to a reflected signal of SNR equal to a) $20 \mathrm{~dB}$ and b) $3 \mathrm{~dB}$ (after Ray 2000).
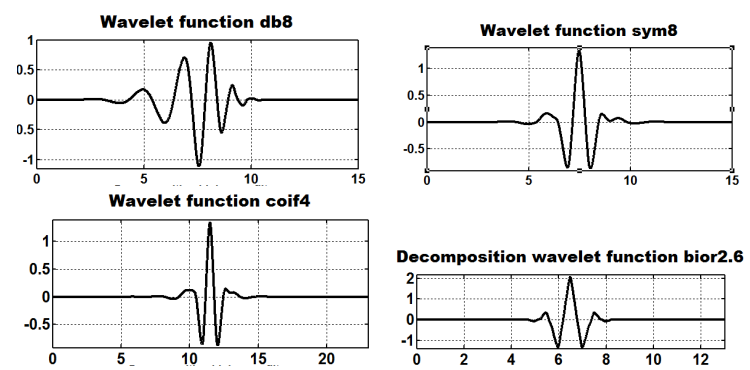

Figure 2. Candidate wavelet base function at different vanishing moments.

there are wavelet subclasses distinguished by the number of coefficients and by the level of iterations. The filter lengths and the number of vanishing moments for four different wavelet families are tabulated in Table 1. For its ability to decompose the signal into independent frequency bands through a nested sequence in the wavelet transform domain, orthogonal wavelets must be selected. The wavelets functions shown in Table 1 are candidates to detect high-frequency error in GPS signals as they can match the sinusoidal and saw-tooth pattern shown in Figure 1.

Table 1. Wavelets families and their properties.

\begin{tabular}{cccc}
\hline $\begin{array}{c}\text { Wavelets } \\
\text { Family }\end{array}$ & $\begin{array}{c}\text { Filter } \\
\text { length }\end{array}$ & $\begin{array}{c}\text { Number of Vanishing Orthogonal } \\
\text { moments }\end{array}$ & \\
\hline \hline Daubechies & $2 \mathrm{M}$ & $\mathrm{M}$ & yes \\
Symlets & $2 \mathrm{M}$ & $\mathrm{M}$ & yes \\
Coiflets & $6 \mathrm{M}$ & $2 \mathrm{M}-1$ & yes \\
Biorthogonal $\max (2 \mathrm{Nr}, 2 \mathrm{Nd})+2$ & $2 \mathrm{M}-1 / 2 \mathrm{M}$ & No \\
\hline
\end{tabular}

\subsection{Level of decomposition}

Multi-Resolution Analysis (MRA) is used in the construction of orthogonal wavelet bases and the fast decomposition of a signal into 


\section{Journal of Geodetic Science}

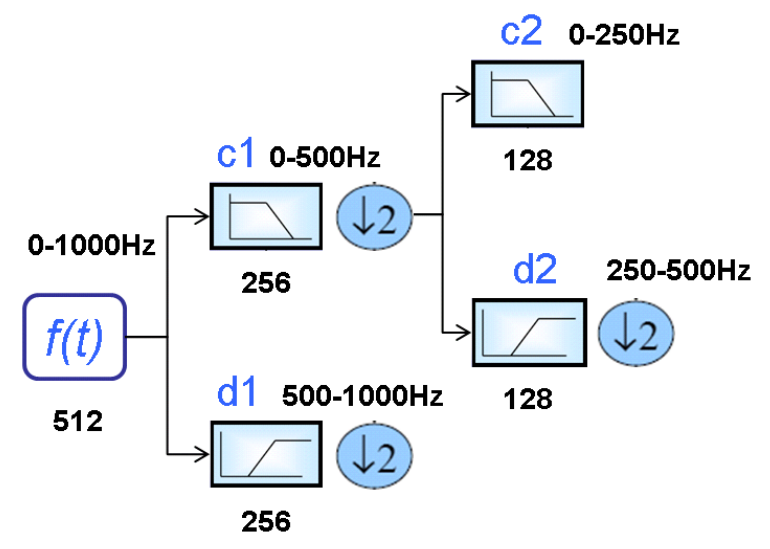

Figure 3. Block diagram of the one-dimensional wavelets decomposition (Elhabiby 2007).

independent frequency bands through a nested sequence. MRA builds a pyramidal structure that requires an iterative application of scaling or levels of decomposition and wavelets functions to low pass (LP) and high pass (HP) filters, respectively (Figure 3). These filters initially act on the entire signal band at a high frequency (lower scale values) and gradually reduce the signal band at each stage. As a result of this structure, the signal is decomposed into an approximation $c_{n}{ }^{m}$ and a detailed $d_{n}{ }^{m}$ part. Decomposition into high level is required to capture the low-frequency multipath. In this research paper an investigation is conducted on the level of decomposition that captures most of the correlated errors between levels 1 to 12 on $1 \mathrm{~Hz}$ GPS double difference data.

\subsection{Threshold type}

The wavelet thresholding technique was developed primarily for removing noise and outliers, compression, and pattern recognition of the signal before wavelets reconstruction. In this analysis, two thresholding methods are presented: hard and soft thresholding. The former is for matrix compression and the latter for de-noising signals.

Hard thresholding is like a gate. If a value is below a certain thresholding value, it is set to zero (Figure 4). Wavelet coefficients (given as an absolute value) larger than a certain specified threshold $\delta$ should be included in the reconstruction. The reconstructed function $\hat{q}(t)$ can be expressed as follows (Ogden 1997):

$$
\hat{q}(t)=\sum_{m} \sum_{n} I_{\left\{\left|d^{m}{ }_{n}\right|>\delta\right\}} d^{m}{ }_{n} \psi_{m, n}
$$

Where $I_{\left\{\left|d^{m}{ }_{n}\right|>\delta\right\}}$ is the indicator function of this set of wavelet coefficients for thresholding, $\psi_{m, n}$ is the wavelets function generated from the original mother wavelets $\psi \in L^{2}(\mathrm{R}), m$ is the scale or level of decomposition, and $n$ is the shifting or translation integer respectively.
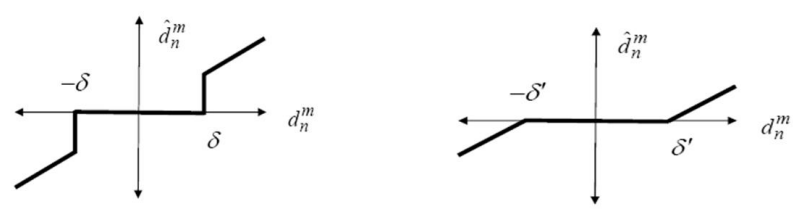

Figure 4. Hard threshold (left) and soft threshold (right).

This function represents a "keep or kill" wavelet reconstruction technique in that it assumes a value of one for the coefficients required in the reconstruction process and zero for the coefficients that should be removed. Hard thresholding is a type of nonlinear operator on the wavelet coefficients vector and leads to a resultant vector of the estimated coefficients $\hat{d}_{n}^{m}$, which can be involved in the reconstruction process, as follows:

$$
\hat{d}_{n}^{m}=\left\{\begin{array}{cl}
d^{m}{ }_{n}, & \text { if }\left|d^{m}{ }_{n}\right| \geq \delta \\
0, & \text { otherwise }
\end{array}\right.
$$

Soft thresholding is defined as

$$
\hat{d}_{n}^{m}=\left\{\begin{array}{cc}
d^{m}{ }_{n}-\delta, & \text { if }\left|d^{m}{ }_{n}\right| \geq \delta \\
0, & \text { if }\left|d^{m}{ }_{n}\right| \leq \delta \\
d^{m}{ }_{n}-\delta, & \text { if }\left|d^{m}{ }_{n}\right|<\delta
\end{array}\right.
$$

Based on Figure 4, it is clear that soft thresholding is generally linear (straight line with slope to be determined). The input for this figure is wavelet coefficient $d^{m}{ }_{n}$ before thresholding and the output is the estimated coefficient $\hat{d}_{n}^{m}$ after thresholding. Soft thresholding is used in de-noising signals hidden in background noise. The main objective is to attenuate the noise while amplifying the signal. The thresholding $\delta^{\prime}$ value is computed using a threshold estimator (Ogden 1997).

\subsection{Threshold estimator}

Choosing a threshold value in the threshold function is a key challenge for in-signal de-noising. The reason for this is that the threshold estimator, which controls the flow of noise in the signal, can have a very small threshold value. This can cause some noise to be saved after the signal de-noising. On the other hand, a larger thresholding value will cause distortion. Therefore, it is crucial to select the proper threshold value while performing the de-noising technique. Donoho and Johnstone (1994) presented several proposals about the choice of the threshold estimators. In this paper four threshold estimators are investigated for GPS error mitigation in the double difference domain, these estimators are:

1- Stein's unbiased maximum likelihood threshold estimator (Rigrsure) 
This estimator uses the SURE threshold, which was established using Stein's unbiased maximum likelihood estimator. For each threshold value the corresponding value-at-risk is found and then the threshold that reduces the risk threshold values is chosen based on the following algorithms:

$$
\mathrm{P}=\left[\mathrm{P}_{0}, \mathrm{P}_{1}, \cdots, \mathrm{P}_{\mathrm{N}-1}\right], \mathrm{P}_{0}<\mathrm{P}_{1}<\cdots<\mathrm{P}_{\mathrm{N}-1}
$$

The elements of $P$ are squares of the wavelets coefficients, which are sorted from small to large order. The risk algorithm is:

$$
R(K)=\left[N-2 K-(N-K) P_{k}+\sum_{I=1}^{k} P_{I}\right] / N
$$

Where $k=0,1, \ldots, N-1$. According to the resulting risk curve $R(k)$, the minimum corresponding value to be $K_{\min }$ and the threshold is defined as:

$$
\lambda=\sigma \sqrt{\mathrm{PK}_{\min }}
$$

2- Median threshold estimator

This threshold value is based on the following wavelet transform:

$$
\lambda=\sigma \sqrt{2 \log N}
$$

Where $N$ is the signal length, $\sigma$ is the noise standard deviation, which can be evaluated by the following equation,

$$
\sigma=\frac{\operatorname{median}\left(\left|\mathrm{d}_{\mathrm{j}}(\mathrm{k})\right|\right)}{0.6745}
$$

Where $d_{j}(k)$, is the high frequency coefficient after the wavelets decomposition, and median is calculating intermediate values of the operation.

3- Heuristic threshold estimator

The heuristic threshold estimator synthesizes the two former thresholds. When the ratio of the signal and noise is small, a fixed threshold is adopted; otherwise, using Rigrsure is the norm.

\section{4- Minimum Maximum (Minimax) threshold estimator}

The Minimax threshold estimator is a thresholding method developed by (Donoho and Johnstone, 1994) that is based on minimizing the 12 risk (Eq. (11)). The minimax principle is used in statistics to design estimators. For unknown regression functions, it is possible to integrate the de-noised signal to the estimator; therefore, the minimax estimator realizes the minimum of the maximum mean square error for the worst function in a certain set.

$$
\begin{aligned}
& \Lambda^{+}{ }_{n} \leq 2 \log n+1 \text { and } \lim _{n \rightarrow \infty} \Lambda^{+}{ }_{n}=2 \log n \\
& {\lambda^{+}}_{n} \equiv \text { the largest } \lambda \text { attaining } \Lambda^{+}{ }_{n} \\
& \Lambda^{+}{ }_{n} \leq \sqrt{2 \log n} \text { and } \lim _{n \rightarrow \infty} \lambda^{+}{ }_{n}=\sqrt{2 \log n}
\end{aligned}
$$
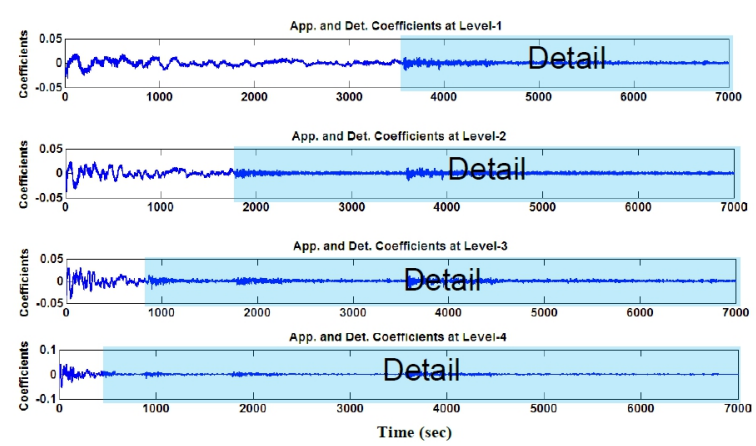

Figure 5. Approximation and details coefficients at different level of decomposition.

\section{Wavelet De-Trending}

The low-frequency portion of multipath is what creates the largest error, which in carrier phase measurements can reach up to $5 \mathrm{~cm}$. Wavelets are used to remove the high-frequency oscillation from the investigated signal by changing the detail coefficient values of the wavelet decomposition to zero and reconstructing the signal using the modified wavelet coefficients. If the details associated with noise cannot be determined properly, either useful signals will be missed or a reconstructed signal may contain severe noise. In the case of a double difference signal, multipath is distributed at varying levels of decomposition. In order to reach the low-frequency multipath error, a higher level of decomposition is required (Time (sec) Figure 5). Thresholding the details coefficients at a level where the largest low-frequency multipath error is suspected will reduce the overall error. But to reach that error, other unwanted frequencies are induced in the reconstructed signal at the lower levels of decomposition.

A new approach based on a wavelet de-trending technique is introduced to remove the long wavelength carrier phase multipath error in the measurements domain. In order to mitigate multipath, GPS double difference observables are introduced to an adaptive wavelets analysis procedure based on high and low pass filter decomposition with varying levels of resolution (El-Ghazouly et al. 2008). The procedure is applied after cycle slips detection and repair. Based on the previous knowledge and facts that the largest errors are caused by the low-frequency multipath, the wavelet transform approach is used to separate the multipath error at high levels of decomposition. The separated wavelet coefficients (approximation or high-level decomposition coefficients) are truncated using wavelets thresholding techniques before the reconstruction of the signal to acquire the true double difference carrier phase residuals out of the low-frequency multipath (El-Ghazouly 2009).

\section{VERSITA}




\section{Case Study of baseline range $100 \mathrm{~m}$ to $50 \mathrm{~km}$}

This section describes GPS data collection campaigns that were conducted between 2007 and 2012 in order to investigate GPS error in the multi-resolution domain and develop the proper procedure for error mitigation. The focus of these experiments was to investigate correlated errors in the double difference measurement, a critical step for building the necessary procedures for mitigation. Three experiments were designed to cover baselines from $100 \mathrm{~m}$ to $50 \mathrm{~km}$ in different geometric conditions. The first experiment was established at the University of Calgary in November 2007 using NovAtel's GPS receivers. It was developed in a controlled environment where very short baselines were used to eliminate ionospheric and tropospheric errors and multipath remained the main source of error. The objective of this experiment was to assess multipath and noise errors that can be introduced in reference stations and then evaluate their characteristics within the multi-resolution domain. To extend the baseline length from $100 \mathrm{~m}$ to $30 \mathrm{~km}$, a second experiment was conducted at Nose Hill Park, in northwest Calgary. In this experiment, two types of receivers were used: Trimble R8 dual-frequency GPS receivers along with an IGS station designated PRDS, near Priddis, southeast of Calgary. The data was collected at four GPS receivers simultaneously between January $9^{\text {th }}$ and $11^{\text {th }} 2008$ at one-second intervals. To further extend the analysis to a $50 \mathrm{~km}$ baseline, GPS observations were collected from the Cansel VRS network (Can-Net) from May 22 ${ }^{\text {nd }}$ to May $24^{\text {th }}, 2012$.

\subsection{Description of Experiments}

\subsubsection{Experiment \#1 (Short baseline at the University of Calgary)}

This experiment is designed under controlled environment to eliminate most of DGPS errors and keep multipath error as the main error source. The ultimate goal of this experiment is to distinguish the multipath signature because of short and long delays, and then transfer it to the multi-resolution domain. This experimental test was made at the University of Calgary using four NovAtel 600 dualfrequency antennas and four OM4 GPS receivers. The data was collected at both the Calgary Center for Innovative Technology (CCIT) and the Engineering building on the University of Calgary campus, shown in Figure 6. The equipment used at each station is listed in Table 2. The configuration at the reference station consisted of one receiver and antenna, which were used to collect data on the roof of the Engineering building.

On the roof of the CCIT building, the three rover stations were arranged, as shown in Figure 6. The data collection started on November $11^{\text {th }}, 2007$ and lasted for three days at a rate of onesecond data intervals. The total number of epochs collected per day was 7200 , which reflects two hours of data collection per day. The baseline length between the reference station and the rover station was in the range of $100 \mathrm{~m}$ so as to guarantee the elimination of all the correlated errors such as ionospheric and tropospheric errors when applying the double difference measurements (Hofmann 2001). The only remaining errors should be the multi-

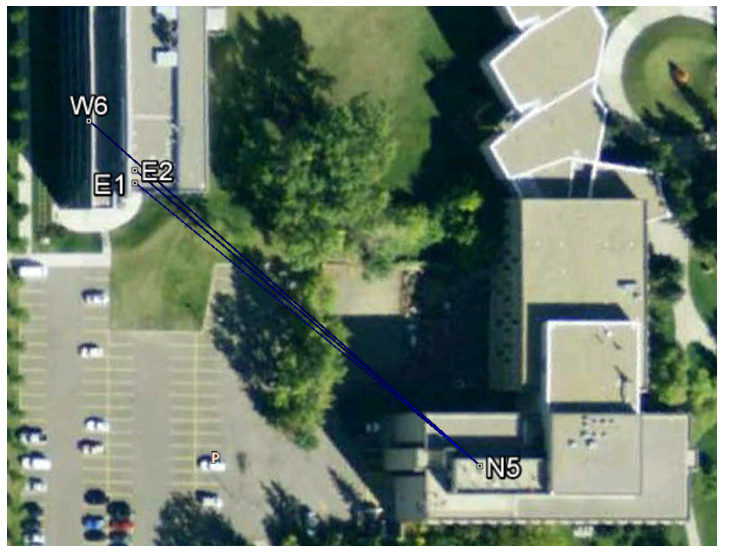

Figure 6. Experiment \#1 Short baselines at university of Calgary.

path and the antenna phase center variation. To minimize antenna phase center error, a NovAtel 600 antenna which has a stable phase center variation was used, and both the reference and rover antennas were oriented to the north direction.

\subsubsection{Experiment \#2 (Short to medium baseline at Nose Hill park)}

This experiment is designed to investigate the behavior of GPS errors in the multi-resolution domain. It was established using four GPS points set on Nose Hill Park in northwest Calgary. Nose Hill Park is a natural environment park, which provides ideal conditions for GPS observations. It allows a clear view of the sky without any obstructions. Those points, designated $\mathrm{NH} 1, \mathrm{NH} 2, \mathrm{NH} 3$, and $\mathrm{NH} 4$ were marked with an iron rebar drilled into the ground. All points were occupied by Trimble R8 dual-frequency GPS receivers mounted on tripods above the markers. A fifth point, N5 used in Experiment \#1, was chosen on the roof of the engineering building at the University of Calgary. In this station, the 600 dual-frequency antennas and OEM4 GPS receivers that were used in the previous experiment were also used again in this experiment. Furthermore, an IGS station designated Prds, near Priddis south-east of Calgary, was used as an additional base station. The experiment was conducted over three days, from January $9^{\text {th }}$ to January $11^{\text {th }}, 2008$. Each receiver was used at the same point on all days. All receivers were operated in static mode. The data collection started on January $9^{\text {th }}, 2008$ and collected on three different days at the rate of one second intervals. The total number of epochs collected per day was 7200 , which reflects two hours of data collection per day. The baseline length ranged from 200 meters between $\mathrm{NH} 1$ and $\mathrm{NH} 3$ to 30.80 kilometers between Prds and NH1. Table 3 shows the receiver used at each station, the dates of data collection and duration.

\subsubsection{Experiment \#3 (Can-Net Medium baseline)}

To assess the proposed multi-resolution technique for mitigation of medium to low frequency errors, GPS observations were col-

\section{VERSITA}


Table 2. Observation dates and equipment used for reference and rover measurements.

\begin{tabular}{|c|c|c|c|c|}
\hline Station & Receiver & Antenna & Observation & $\frac{\text { Duration }}{\text { (Hour) }}$ \\
\hline \multirow{3}{*}{ E1, E2, W6 } & \multirow{3}{*}{ Probak-DL-4-RT2 Novatel, } & \multirow{3}{*}{ GPS-600-LB } & 11-Nov Day01 & 2 \\
\hline & & & 12-Nov Day02 & 2 \\
\hline & & & 13-Nov Day03 & 2 \\
\hline \multirow{3}{*}{ N5 } & \multirow{3}{*}{ Probak-G2-DB9-RT2 Novatel, } & \multirow{3}{*}{ GPS-600-LB } & 11-Nov Day01 & 2 \\
\hline & & & 12-Nov Day02 & 2 \\
\hline & & & 13-Nov Day03 & 2 \\
\hline
\end{tabular}

Table 3. Observation dates and equipment used for data collection in experiment \#02.

\begin{tabular}{ccccc}
\hline & Receiver Info & & \multirow{2}{*}{ Observation } & \multirow{2}{*}{ Duration (Hour) } \\
\hline PtID & Receiver Type & ReclD & & \\
\hline \hline NH1 & Trimble R8-2 & 4651126518 & & 2 \\
\hline NH2 & Trimble R8-2 & 4629118870 \\
\hline NH3 & Trimble R8-2 & 4629118867 \\
\hline NH4 & Novatel Probak & 1017201 \\
\hline N5 & Novatel Probak & 1017062 \\
\hline
\end{tabular}

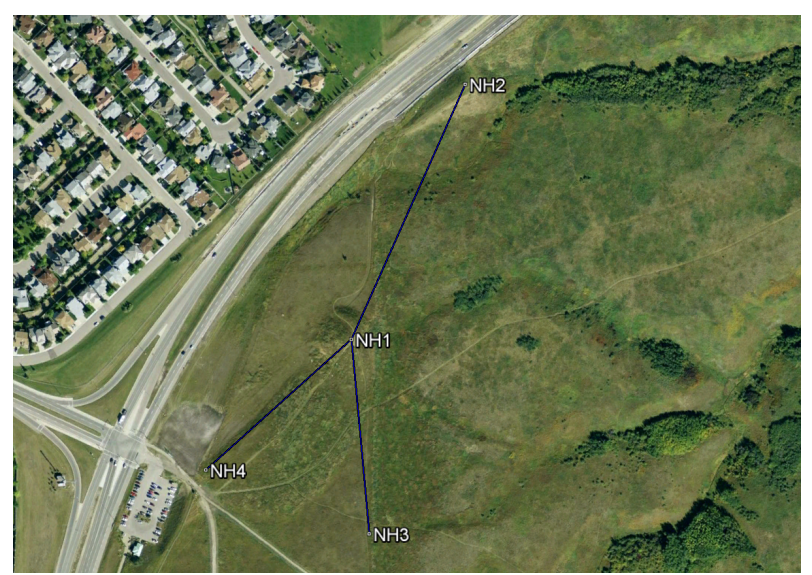

Figure 7. Experiment \#2 Short baselines at Nose Hill Park.

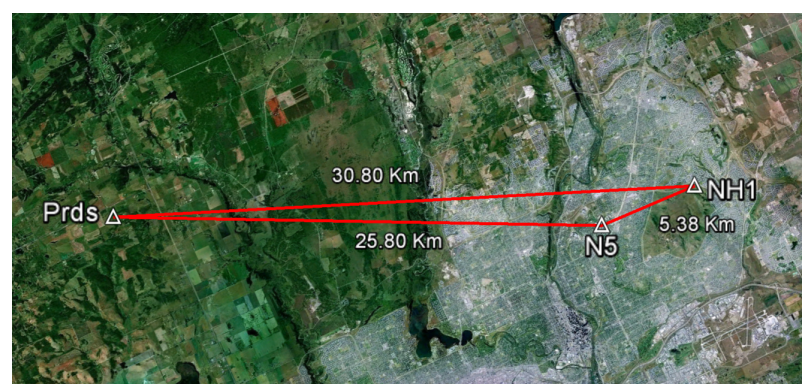

Figure 8. Experiment \#2 longer baseline from NH1 to N5 and PRDS.

VERSITA

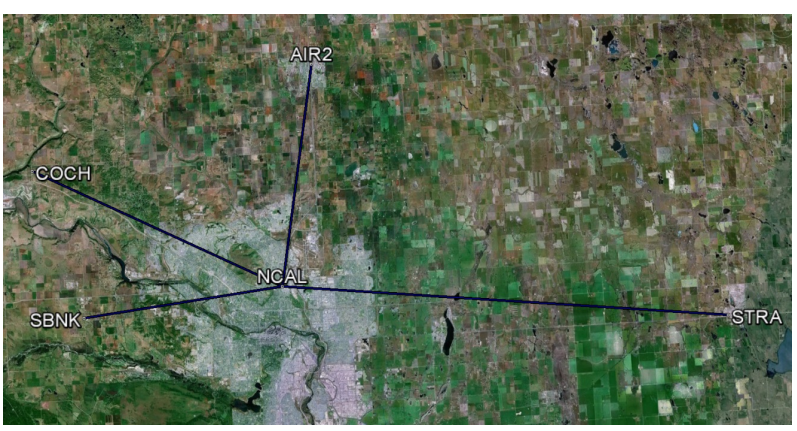

Figure 9. Experiment \#3 Can-Net stations.

lected from the Cansel VRS network (Can-Net). The Canada Network (Can-Net) consists of 260 GPS receivers across Canada, deployed in 2005 by the Cansel Survey Equipment. Five Can-Net stations are used in this paper (Figure 9) to cover baselines from $23 \mathrm{~km}$ to $50 \mathrm{~km}$. Each Can-Net station consists of a Trimble Zephyr Geodetic 2 antenna and either Trimble NetR5 or Trimble NetR9 receiver (Table 4). The data was collected over three days for two hours per day at one second intervals beginning on May $22^{\text {nd }}, 2012$.

\subsection{Computation of Reference Coordinates}

Data collected in the above three experiments was first processed using Bernese 4.2 (Hugentobler, 2001). Different techniques were used in Bernese software to compute the coordinates for the data collected in this research paper. The coordinates shown in Table 5 
Table 4. Observation dates and equipment used for data collection in experiment \#03.

\begin{tabular}{|c|c|c|c|c|c|}
\hline \multirow{2}{*}{ STN Name } & \multicolumn{3}{|c|}{ Receiver/ Antenna type } & \multirow{2}{*}{ Observation } & \multirow{2}{*}{ Duration (Hour) } \\
\hline & Code & Receiver & Antenna & & \\
\hline NCAL & Aref & TRIMBLE NETR9 & & & 2 \\
\hline AIR2 & Ant1 & TRIMBLE NETR9 & & & 2 \\
\hline $\mathrm{COCH}$ & Ant3 & TRIMBLE NETR9 & Trimble Zephyr Geodetic 2 & May $22^{\text {nd }}-$ May $24^{\text {th }}, 2012$ & 2 \\
\hline SBNK & Ant7 & TRIMBLE NETR5 & & & 2 \\
\hline STRA & Ant8 & TRIMBLE NETR5 & & & 2 \\
\hline
\end{tabular}

and Table 6 are computed based on the ionosphere-free fixed solution, $15^{\circ}$ cut-off angle, IGS precise orbit. The model and mapping function for troposphere were the Saastamoinen model and the $\cos (\mathrm{z})$ mapping function as recommended by Tait et al., 2008.

Table 5. Reference coordinates for experiment \#1 and \#2 computed by Bernese software.

\begin{tabular}{ccccc}
\hline Station & X & Y & Z & Baseline \\
\hline \hline E1 & -1641937.391 & -3664810.674 & 4940009.495 & 92.474 \\
E2 & -1641936.566 & -3664808.83 & 4940011.13 & 94.142 \\
W6 & -1641942.081 & -3664797.661 & 4940017.63 & 108.118 \\
NH01 & -1640032.815 & -3661204.911 & 4943430.698 & 0 \\
N5 & -1641890.232 & -3664880.419 & 4939971.243 & 5378.438 \\
NH02 & -1639847.077 & -3661067.638 & 4943584.182 & 277.342 \\
NH03 & -1640076.321 & -3661345.647 & 4943304.394 & 194.034 \\
NH04 & -1640204.237 & -3661236.241 & 4943348.08 & 192.780 \\
PRDS & -1659602.829 & -3676725.754 & 4925493.591 & $30,750.776$ \\
\hline
\end{tabular}

Table 6. Reference coordinates for experiment \#3 computed by Bernese software

\begin{tabular}{|c|c|c|c|c|}
\hline \multirow{2}{*}{ Station } & \multicolumn{3}{|c|}{ REFERENCE } & \multirow{2}{*}{ BASELINE } \\
\hline & $X$ & $\mathrm{Y}$ & Z & \\
\hline NCAL & -1636751 & -3666214.588 & 4940590.545 & 0.00 \\
\hline AIR2 & -1625733 & -3651749.881 & 4954880.696 & $23,126.57$ \\
\hline $\mathrm{COCH}$ & -1655669 & -3646664.28 & 4949021.922 & $28,481.19$ \\
\hline SBNK & -1656244 & -3659412.647 & 4939286.997 & $20,686.16$ \\
\hline STRA & -1596479 & -3688603.493 & 4937025.199 & $46,215.15$ \\
\hline
\end{tabular}

Table 7 summarizes the baselines used in the analysis and the number of double difference data for each baseline. The baselines are divided into two categories according to the baseline length. Category I contains short baselines where the length varies from $100 \mathrm{~m}$ to $5 \mathrm{~km}$, and Category II covers longer baselines from $20 \mathrm{~km}$ to $50 \mathrm{~km}$. The table also shows each baseline reference and rover station, the number of double differenced data computed in this baseline and the reference satellite used to compute the double difference data.
The total number of baselines in these data sets is 13 with seven baselines in Category I, and six baselines in Category II. In this research case study the double difference measurements are computed in L1 GPS signal and ionosphere-free linear combination. Data was collected over three days for approximately two hours for each baseline. It is worth mentioning that three successive days of GPS observations for each baseline were used in this paper to identify correlated errors. Moreover, only the satellites available through the 7200 epochs were used to generate double difference measurements in this paper.

Table 7. Baselines and double difference data.

\begin{tabular}{|c|c|c|c|c|c|}
\hline Category & Ref & Rover & Baseline & No. of DDs. & Ref. Sat. \\
\hline \multirow{7}{*}{ I } & N5 & E1 & 92.5 & 13 & 2 \\
\hline & N5 & E2 & 94.1 & 13 & 2 \\
\hline & N5 & W6 & 108.1 & 12 & 2 \\
\hline & $\mathrm{NH} 1$ & $\mathrm{NH} 2$ & 277.3 & 13 & 30 \\
\hline & $\mathrm{NH} 1$ & $\mathrm{NH3}$ & 194.0 & 13 & 30 \\
\hline & $\mathrm{NH} 1$ & $\mathrm{NH} 4$ & 192.8 & 13 & 30 \\
\hline & $\mathrm{NH} 1$ & N05 & $5,378.4$ & 11 & 30 \\
\hline \multirow{7}{*}{ II } & $\overline{N C A L}$ & SBNK & $20,686.2$ & 7 & 28 \\
\hline & NCAL & AIR2 & $23,126.6$ & 7 & 28 \\
\hline & N05 & PRDS & $25,756.3$ & 7 & 17 \\
\hline & NCAL & $\mathrm{COCH}$ & $28,481.2$ & 7 & 17 \\
\hline & $\mathrm{NH} 1$ & PRDS & $30,750.8$ & 6 & 30 \\
\hline & NCAL & STRA & $46,215.2$ & 6 & 28 \\
\hline & \multicolumn{3}{|c|}{ Total } & 128 & \\
\hline
\end{tabular}

\subsection{Wavelets multipath mitigation}

\subsubsection{Wavelet base function and vanishing moment}

Thirty-seven wavelet base functions are used to decompose the double difference data into their details and approximation coefficients (Table 8). Each of the 128 double difference measurements mentioned in Table 7 is decomposed to its approximation and details at levels of decomposition from one to 12. The criteria for selecting the best wavelets base candidate are based on a correlation between days. This is explained in the following steps:

1) Compute the correlation for each baseline over the three days of data collection using Eq. (13). There should be 
three reference correlation values for each baseline reflecting the correlation between Day1 and Day2, Day1 and Day3, and Day2 and Day3 for this baseline, which are $C_{12}, C_{13}$ and $C_{23}$.

$$
c_{X Y}=\frac{E\left[\left(X-m_{X}\right)\left(Y-m_{Y}\right)\right]}{\sqrt{E\left[\left(X-m_{X}\right)^{2}\right] E\left[\left(Y-m_{Y}\right)^{2}\right]}}
$$

Where, $E[]$ is the expectation operator, $X$ is a state variable (double difference data on Day 1 ), $Y$ is another state variable (double difference data on Day2), $m_{X}$ is the mean of state variable $X$, and $m_{Y}$ is the mean of state variable $Y$.

2) Decompose each of the 128 double difference data for day one,two and three to approximation and details at Level one. The total number of double difference data in this step is calculated as: 128 double difference data $x$ three days $=$ 384 double difference data.

3) At this level of decomposition set the details coefficients to zero, this step will delete all the high-frequency data at this level.

4) Reconstruct the double difference data from the modified details and approximations.

5) Re-compute the correlation based on Eq. (13) for each modified double difference which are $C_{12}{ }^{\prime}, C_{13}$ 'and $C_{23}$ '.

6) Compute the correlation reduction for each baseline over two days as follows:

$$
c_{\text {red }}=\frac{C^{\prime}-C}{C}
$$

7) Repeat steps 2 through 6 for levels of decomposition 2 to 12.

At this point there should be 128 modified double differences over three days with 12 levels of decomposition. This equals 4608 correlation values. The wavelet base function that reports the highest correlation reduction in these 4608 correlation values is considered the best candidate for this analysis. This is because the wavelet base function that reports the highest correlation reduction is the one that can most efficiently isolate the correlated signals at the wavelets bandwidth to its original details.

Table 9 shows the wavelet base function and the number of times that it reported the maximum correlation reduction. It also shows the frequency with which each wavelet base function reports a maximum correlation reduction for each baseline category in L1 and ionosphere-free measurement in L3. It can be seen from the table that a biorthogonal wavelets family achieved the highest degree of correlation reduction $(60 \%-70 \%)$. The closest family is the
Daubechies family $(25 \%-30 \%)$. It is evident from the figure that the bior2.2 in the biorthogonal wavelets family performed better than all the other wavelets functions for short baselines in Category I, while bior3.3 performed better for longer baselines in Category II. Coiflets and Symlet performed the worst in detecting the correlated signal as they only show around 1\% for Coiflets and 8\% for Symlet.

Moreover, the effect of the base function on both L1 and L3 measurements is almost the same except for longer baselines (Category II). This is the result of ionosphere-free linear combination, which removes the first order ionospheric (uncorrelated errors) errors and makes the correlated error more clear. Consequently, the L3 Category has a higher percentage than the L1 Category, particularly at the biorthogonal family.

Based on this analysis, the use of Coiflets or Symlet is not recommended for correlated error detection of GPS double difference measurements. However, the biorthogonal method is recommended to isolate the correlated error, especially bior 2.2 for short baseline and bior3.3 for longer baselines.

\subsubsection{Level of decomposition}

In this section the level of decomposition that achieves the maximum correlation between days is considered best level of decomposition to be used throughout this paper. Each of the 128 double difference measurements mentioned in Table 7 is decomposed to approximation and details at levels of decompositions from one to 12 using a the bior3.3 base function. The criterion for selecting the best level of decomposition is the same as described in the previous section with the exception of bior3.3, which is the only base function used in this section. There were 128 double difference data sets over three days (a total of 384) that were decomposed using a bior3.3 wavelet base function. Each of these 384 double difference data sets were processed as described in the following steps:

1) Decompose each of the 384 double difference data sets to approximation and details at levels of decomposition from 1 to 12 .

2) At each level of decomposition, set the details coefficients to zero. This step will delete all the high-frequency data at this level.

3) Reconstruct the double difference data from the modified details and approximations.

4) Re-compute the correlation based on Eq. (13) for each modified double difference which are $C_{12}{ }^{\prime}, C_{13}{ }^{\prime}$ and $C_{23}{ }^{\prime}$.

5) Compute the correlation reduction for each baseline between two days using the reference correlation values computed in the previous section.

6) For each baseline at each day, record the level of decomposition that provides the maximum correlation reduction. 
Table 8. Candidates wavelet base function.

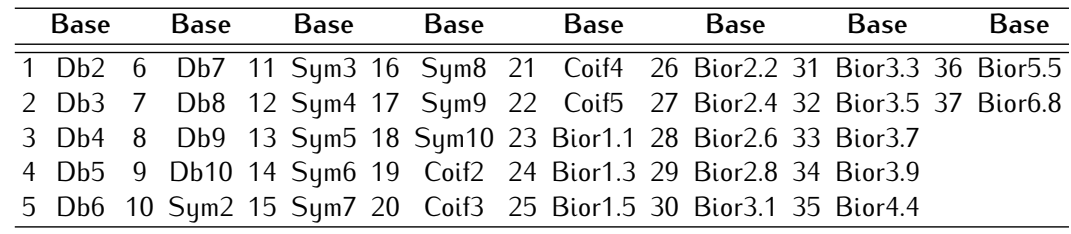

Table 9. Wavelet base function and the number of times it recorded the maxiumuin Correlation reduction.

\begin{tabular}{|c|c|c|c|c|c|c|c|c|c|c|c|c|}
\hline & \multicolumn{3}{|c|}{ L1_Category I } & \multicolumn{3}{|c|}{ L1_Category II } & \multicolumn{3}{|c|}{ L3_Category I } & \multicolumn{3}{|c|}{ L3_Category II } \\
\hline & $\mathrm{N}$ & $\%$ & Total & $\mathrm{N}$ & $\%$ & Total & $\mathrm{N}$ & $\%$ & Total & $\mathrm{N}$ & $\%$ & Total \\
\hline $\mathrm{db} 2$ & 432 & 13.6 & & 161 & 11.2 & & 442 & 14.0 & & 182 & 12.6 & \\
\hline db3 & 101 & 3.2 & & 23 & 1.6 & & 67 & 2.1 & & 32 & 2.2 & \\
\hline $\mathrm{db} 4$ & 105 & 3.3 & & 48 & 3.3 & & 107 & 3.4 & & 45 & 3.1 & \\
\hline db5 & 69 & 2.2 & & 19 & 1.3 & & 66 & 2.1 & & 11 & 0.8 & \\
\hline db6 & 54 & 1.7 & 30.2 & 17 & 1.2 & 24.0 & 42 & 1.3 & 28.4 & 15 & 1.0 & 231 \\
\hline db7 & 47 & 1.5 & & 15 & 1.0 & & 40 & 1.3 & & 13 & 0.9 & \\
\hline db8 & 51 & 1.6 & & 23 & 1.6 & & 48 & 1.5 & & 14 & 1.0 & \\
\hline db9 & 44 & 1.4 & & 19 & 1.3 & & 35 & 1.1 & & 10 & 0.7 & \\
\hline $\mathrm{db} 10$ & 54 & 1.7 & & 21 & 1.5 & & 53 & 1.7 & & 11 & 0.8 & \\
\hline sym 4 & 78 & 2.5 & & 26 & 1.8 & & 66 & 2.1 & & 21 & 1.5 & \\
\hline sym 5 & 43 & 1.4 & & 10 & 0.7 & & 38 & 1.2 & & 11 & 0.8 & \\
\hline sym6 & 24 & 0.8 & & 5 & 0.3 & & 13 & 0.4 & & 3 & 0.2 & \\
\hline sym7 & 54 & 1.7 & 8.5 & 16 & 1.1 & 5.6 & 48 & 1.5 & 6.8 & 13 & 0.9 & 3.8 \\
\hline sym 8 & 10 & 0.3 & & 5 & 0.3 & & 2 & 0.1 & & 2 & 0.1 & \\
\hline sym 9 & 34 & 1.1 & & 11 & 0.8 & & 24 & 0.8 & & 2 & 0.1 & \\
\hline sym10 & 25 & 0.8 & & 7 & 0.5 & & 23 & 0.7 & & 2 & 0.1 & \\
\hline coif2 & 14 & 0.4 & & 0 & 0.0 & & 12 & 0.4 & & 3 & 0.2 & \\
\hline coif3 & 12 & 0.4 & & 0 & 0.0 & & 8 & 0.3 & & 2 & 0.1 & \\
\hline coif4 & 19 & 0.6 & 2.6 & 0 & 0.0 & 0.8 & 22 & 0.7 & 2.1 & 3 & 0.2 & 0.8 \\
\hline coif5 & 38 & 1.2 & & 12 & 0.8 & & 23 & 0.7 & & 3 & 0.2 & \\
\hline bior1.1 & 0 & 0.0 & & 0 & 0.0 & & 0 & 0.0 & & 0 & 0.0 & \\
\hline bior1.3 & 249 & 7.9 & & 4 & 0.3 & & 211 & 6.7 & & 120 & 8.3 & \\
\hline bior1.5 & 82 & 2.6 & & 106 & 7.4 & & 62 & 2.0 & & 35 & 2.4 & \\
\hline bior2.2 & 492 & 15.5 & & 33 & 2.3 & & 539 & 17.0 & & 273 & 19.0 & \\
\hline bior2.4 & 62 & 2.0 & & 264 & 18.3 & & 50 & 1.6 & & 16 & 1.1 & \\
\hline bior2.6 & 26 & 0.8 & & 21 & 1.5 & & 16 & 0.5 & & 7 & 0.5 & \\
\hline bior2.8 & 27 & 0.9 & & 8 & 0.6 & & 21 & 0.7 & & 5 & 0.3 & \\
\hline bior3.1 & 294 & 9.3 & & 7 & 0.5 & & 497 & 15.7 & & 340 & 23.6 & \\
\hline bior3.3 & 383 & 12.1 & 58.1 & 298 & 20.7 & 69.6 & 365 & 11.5 & 02.8 & 177 & 12.3 & .4 \\
\hline bior3.5 & 100 & 3.2 & & 192 & 13.3 & & 114 & 3.6 & & 36 & 2.5 & \\
\hline bior3.7 & 49 & 1.5 & & 28 & 1.9 & & 32 & 1.0 & & 7 & 0.5 & \\
\hline bior3.9 & 30 & 0.9 & & 12 & 0.8 & & 34 & 1.1 & & 8 & 0.6 & \\
\hline bior4.4 & 25 & 0.8 & & 12 & 0.8 & & 16 & 0.5 & & 7 & 0.5 & \\
\hline bior5.5 & 31 & 1.0 & & 11 & 0.8 & & 21 & 0.7 & & 6 & 0.4 & \\
\hline bior6.8 & 10 & 0.3 & & 6 & 0.4 & & 11 & 0.3 & & 5 & 0.3 & \\
\hline Total & 31 & 68 & 100.0 & 14 & 140 & 100.0 & & 68 & 100.0 & & 140 & 100.0 \\
\hline
\end{tabular}

Table 10 shows the frequency with which the maximum correlation reduction is achieved for each level of decomposition. It can be seen from the table that Level 1 and 2 are not shown in the table as they never indicate any maximum correlation reduction. This is because Level 1 and 2 contain mostly uncorrelated white noise. From Level 3 to Level 5 , there is only $1 \%-2 \%$ of the maximum cor- relation detected, which is a strong indication that the correlated signal did not appear until Level 5. Levels 6 and 7 indicate a maximum correlation of $10 \%$ of the time. Most of the correlated signal appeared at Levels 8, 9 and 10. For Category I baselines (short baselines), Levels 8 and 9 have the most correlated signal, while Category II baselines (longer baselines) indicate most of the correlation 
at Levels 9 and 10. The results from L1 measurements are identical to the results from $L 3$ measurements, which is a strong indication that the level of decomposition is acting the same in both L1 and L3 measurements.

\subsubsection{Threshold type and threshold estimator}

Each of the 128 double difference measurements mentioned in Table 7 were decomposed to approximation and details at levels of decomposition from one to 12 using bior3.3. The criterion for selecting the best wavelets base candidate is described in the wavelet base function and vanishing moment section. The difference in this section is that only one base function (bior3.3) was used. Table 11 shows the frequency with which the maximum correlation reduction is achieved for each thresholding type/estimator. It can be seen from the table that Median_s, where $\mathrm{s}$ stands for soft and $\mathrm{h}$ for hard thresholding, shows approximately $50 \%$ of the maximum correlation reduction and the second best type/estimator Minimax_s with around 30\%. These results were for baselines for categories I and II and for L1 and L3.

\subsection{Results}

\subsubsection{Short Baselines}

The first category of baselines, of a few hundred meters to five kilometres, was used to compute the coordinates for E1, E2, W6, NH2, $\mathrm{NH} 3, \mathrm{NH} 4$ and NH5. Table 12 shows the coordinate differences (bias) and the root mean square error of these coordinates with respect to the reference coordinates by employing the Bernese software. The results for the short baseline match the reference coordinates within $5-15 \mathrm{~mm}$ and RMS within $10 \mathrm{~mm}$. In this category, the L1 fixed solution is used, as the correlated errors are either eliminated or radically removed in the short baselines. Wavelets Multi-Resolution is applied to the L1 measurement using both the median threshold estimator and the kill approximation techniques. The bior 3.3 wavelet base function is used to decompose each double difference measurement to eight levels of decomposition. The proposed wavelet de-trending technique is then used to separate and differentiate different frequencies from high to low, corresponding with GPS errors. This produced a corrected double difference data as explained in the previous section. In addition, the performance of the wavelet de-trending technique is compared to the traditional wavelet de-noising method using a median estimator.

The corrected double difference measurements from both denoising and de-trending techniques are used with the least square adjustment to produce a fixed GPS solution. The Least-squares AMBiguity Decorrelation Adjustment (LAMBDA) method was used to fix the ambiguity (Teunissen, 1993) in addition to the Saastamoinen model for troposphere error modeling. Figure 10 and Figure 11 show the bias and RMS reduction in the final solution $(X, Y$ and $Z$ components). The double difference measurements were corrected using both de-trending and de-noising techniques. It

\section{VERSITA}

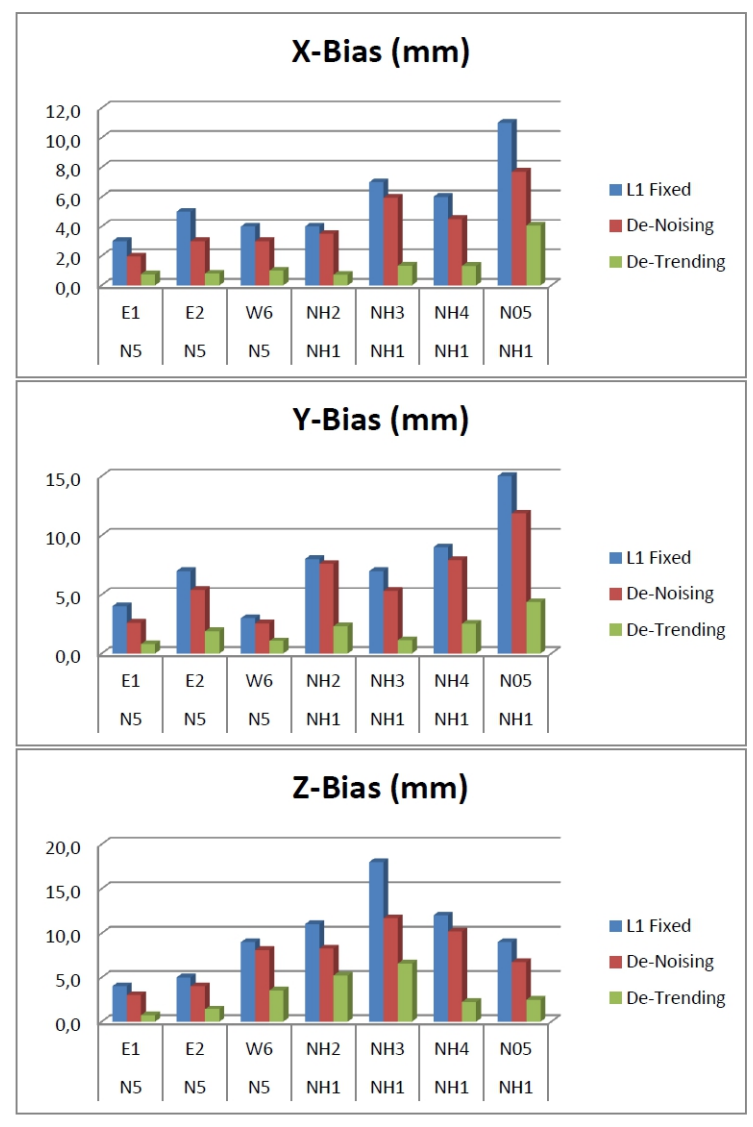

Figure 10. Coordinate bias when computed with raw data (L1 Fixed), De-Noising and De-Trending techniques for short baselines.

can be seen from Figure 10 that the de-noising technique reduced the bias for short baselines from $5 \%$ at X-Bias for $\mathrm{NH} 1-\mathrm{NH} 2$ baseline (from $8 \mathrm{~mm}$ to $7.6 \mathrm{~mm}$ ) to $40 \%$ at X-Bias for N5-E2 baseline (from $5 \mathrm{~mm}$ to $3 \mathrm{~mm}$ ). The RMS improvements for short baselines vary between $16 \%$ at Y-RMS from baseline N5-E1 (from $8 \mathrm{~mm}$ to $6.7 \mathrm{~mm}$ ) to $44 \%$ at X-RMS for baseline NH1-N5 (from $7 \mathrm{~mm}$ to $3.9 \mathrm{~mm}$ ). The average bias reduction for all the $X, Y$ and $Z$ components is $23 \%$, while the average RMS reduction is $30 \%$ when using the de-noising technique (Figure 11). The de-trending technique reduces the bias and RMS as shown in Figure 10 and Figure 11. The bias for short baselines is reduced from $53 \%$ at Z-Bias for $\mathrm{NH} 1-\mathrm{NH} 2$ baseline (from $11 \mathrm{~mm}$ to $5.2 \mathrm{~mm}$ ) to $84 \%$ at X-Bias for N5-E2 baseline (from $5 \mathrm{~mm}$ to $0.8 \mathrm{~mm}$ ). The RMS reductions for short baselines vary between $51 \%$ at Y-RMS from baseline N5-W6 (from $13 \mathrm{~mm}$ to $6.3 \mathrm{~mm}$ ) to $82.5 \%$ at $Y$-RMS for baseline N5-E2 (from $8 \mathrm{~mm}$ to $1.4 \mathrm{~mm}$ ). The average bias reduction for all the $X, Y$ and $Z$ components is $74 \%$, while the average RMS reduction is $69 \%$ when using the de-noising technique. 
Table 10. level of decomposition and the number of times it recorded the maximum Correlation reduction.

\begin{tabular}{ccccccccc}
\hline & \multicolumn{7}{c}{ L1_Category I L1_Category II } & L3_Category I L_Category II \\
\cline { 2 - 9 } & $\mathrm{N}$ & $\%$ & $\mathrm{~N}$ & $\%$ & $\mathrm{~N}$ & $\%$ & $\mathrm{~N}$ & $\%$ \\
\hline \hline 3 & 2 & 0.8 & 0 & 0.0 & 2 & 0.8 & 0 & 0.0 \\
4 & 5 & 1.9 & 0 & 0.0 & 5 & 1.9 & 0 & 0.0 \\
5 & 4 & 1.5 & 0 & 0.0 & 4 & 1.5 & 0 & 0.0 \\
6 & 32 & 12.1 & 4 & 3.3 & 32 & 12.1 & 4 & 3.3 \\
7 & 22 & 8.3 & 0 & 0.0 & 22 & 8.3 & 0 & 0.0 \\
8 & 99 & 37.5 & 12 & 10.0 & 99 & 37.5 & 12 & 10.0 \\
9 & 72 & 27.3 & 42 & 35.0 & 72 & 27.3 & 42 & 35.0 \\
10 & 28 & 10.6 & 62 & 51.7 & 28 & 10.6 & 62 & 51.7 \\
\hline Total & 264 & & 120 & & 264 & & 120 \\
\hline
\end{tabular}

Table 11. Threshold type/estimator and the number of times it recorded the maximum Correlation reduction.

\begin{tabular}{|c|c|c|c|c|c|c|c|c|}
\hline & \multicolumn{2}{|c|}{ L1_Category } & \multicolumn{2}{|c|}{ L1_Category } & \multicolumn{4}{|c|}{ L3_Category I L3_Category II } \\
\hline & $\mathrm{N}$ & $\%$ & $\mathrm{~N}$ & $\%$ & $\mathrm{~N}$ & $\%$ & $\mathrm{~N}$ & $\%$ \\
\hline Heuristic_h & 126 & 4.0 & 101 & 7.0 & 145 & 4.6 & 88 & 6.1 \\
\hline Heuristic_s & 75 & 2.4 & 89 & 6.2 & 72 & 2.3 & 91 & 6.3 \\
\hline Minimax_h & 71 & 2.2 & 57 & 4.0 & 89 & 2.8 & 40 & 2.8 \\
\hline Minimax_s & 1090 & 34.4 & 526 & 36.5 & 1152 & 36.4 & 564 & 39.2 \\
\hline Median_h & 38 & 1.2 & 19 & 1.3 & 59 & 1.9 & 30 & 2.1 \\
\hline Median_s & 1630 & 51.5 & 599 & 41.6 & 1566 & 49.4 & 588 & 40.8 \\
\hline Rigrsure_h & 38 & 1.2 & 17 & 1.2 & 39 & 1.2 & 14 & 1.0 \\
\hline Rigrsure _s & 100 & 3.2 & 32 & 2.2 & 46 & 1.5 & 25 & 1.7 \\
\hline Total & & 68 & & 440 & & 58 & & 440 \\
\hline
\end{tabular}

Table 12. Coordinates difference and RMS in mm for short baseline with respect to the coordinates computed using Bernese.

\begin{tabular}{cccccccccccc}
\hline Ref & Rover & Baseline M & Sol & X Bias & Y Bias & H Bias & Z Bias & X RMS & Y RMS H RMS Z RMS \\
\hline \hline N5 & E1 & 92.5 & Fixed & 3.0 & 4.0 & 5.0 & 4.0 & 4.0 & 8.0 & 8.9 & 6.0 \\
N5 & E2 & 94.1 & Fixed & 5.0 & 7.0 & 8.6 & 5.0 & 8.0 & 8.0 & 11.3 & 8.0 \\
N5 & W6 & 108.1 & Fixed & 4.0 & 3.0 & 5.0 & 9.0 & 11.0 & 13.0 & 17.0 & 13.0 \\
NH1 & NH2 & 277.3 & Fixed & 4.0 & 8.0 & 8.9 & 11.0 & 3.0 & 5.0 & 5.8 & 5.0 \\
NH1 & NH3 & 194.0 & Fixed & 7.0 & 7.0 & 9.9 & 18.0 & 5.0 & 9.0 & 10.3 & 9.0 \\
NH1 & NH4 & 192.8 & Fixed & 6.0 & 9.0 & 10.8 & 12.0 & 4.0 & 6.0 & 7.2 & 6.0 \\
NH1 & N05 & 5378.4 & Fixed & 11.0 & 15.0 & 18.6 & 9.0 & 7.0 & 12.0 & 13.9 & 13.0 \\
\hline
\end{tabular}

Table 13. Coordinates difference and RMS in mm for long baseline with respect to the coordinates computed using Bernese.

\begin{tabular}{cccccccccccc}
\hline Ref & Rover & Baseline $\mathrm{m}$ & Sol & \multicolumn{1}{c}{ X Bias } & Y Bias & H Bias & Z Bias & X RMS & Y RMS H RMS Z RMS \\
\hline \hline NCAL SBNK & 20686.2 & Float L1 & 19.0 & 22.0 & 29.1 & 24.0 & 32.0 & 28.0 & 42.5 & 28.0 \\
NCAL AIR2 & 23126.6 & Float L1 & 22.0 & 25.0 & 33.3 & 33.0 & 41.0 & 49.0 & 63.9 & 41.0 \\
N05 & PRDS & 25756.3 & Float L1 & 32.0 & 28.0 & 42.5 & 35.0 & 43.0 & 58.0 & 72.2 & 81.0 \\
NCAL COCH & 28481.2 & Float L1 & 15.0 & 18.0 & 23.4 & 28.0 & 53.0 & 32.0 & 61.9 & 45.0 \\
NH1 & PRDS & 30750.8 & Float L1 & 28.0 & 23.0 & 36.2 & 37.0 & 44.0 & 35.0 & 56.2 & 63.0 \\
NCAL STRA & 46215.2 & Float L1 & 35.0 & 42.0 & 54.7 & 48.0 & 28.0 & 52.0 & 59.1 & 77.0 \\
\hline
\end{tabular}


Table 14. Coordinates difference and RMS in mm for long baseline with respect to the coordinates computed using Bernese.

\begin{tabular}{cccccccccccc}
\hline Ref & Rover & Baseline $\mathrm{m}$ & Sol & X Bias & Y Bias & H Bias & Z Bias & X RMS & Y RMS H RMS Z RMS \\
\hline \hline NCAL SBNK & 20686.2 & Float IF & 4.0 & 3.0 & 5.0 & 9.0 & 12.0 & 14.0 & 18.4 & 8.0 \\
NCAL AIR2 & 23126.6 & Float IF & 2.0 & 3.0 & 3.6 & 12.0 & 10.0 & 9.0 & 13.5 & 16.0 \\
N05 & PRDS & 25756.3 & Float IF & 5.0 & 4.0 & 6.4 & 11.0 & 14.0 & 11.0 & 17.8 & 18.0 \\
NCAL COCH & 28481.2 & Float IF & 8.0 & 10.0 & 12.8 & 8.0 & 17.0 & 20.0 & 26.2 & 20.0 \\
NH1 & PRDS & 30750.8 & Float IF & 6.0 & 7.0 & 9.2 & 15.0 & 11.0 & 17.0 & 20.2 & 63.0 \\
NCAL STRA & 46215.2 & Float IF & 12.0 & 9.0 & 15.0 & 14.0 & 21.0 & 19.0 & 28.3 & 23.0 \\
\hline
\end{tabular}

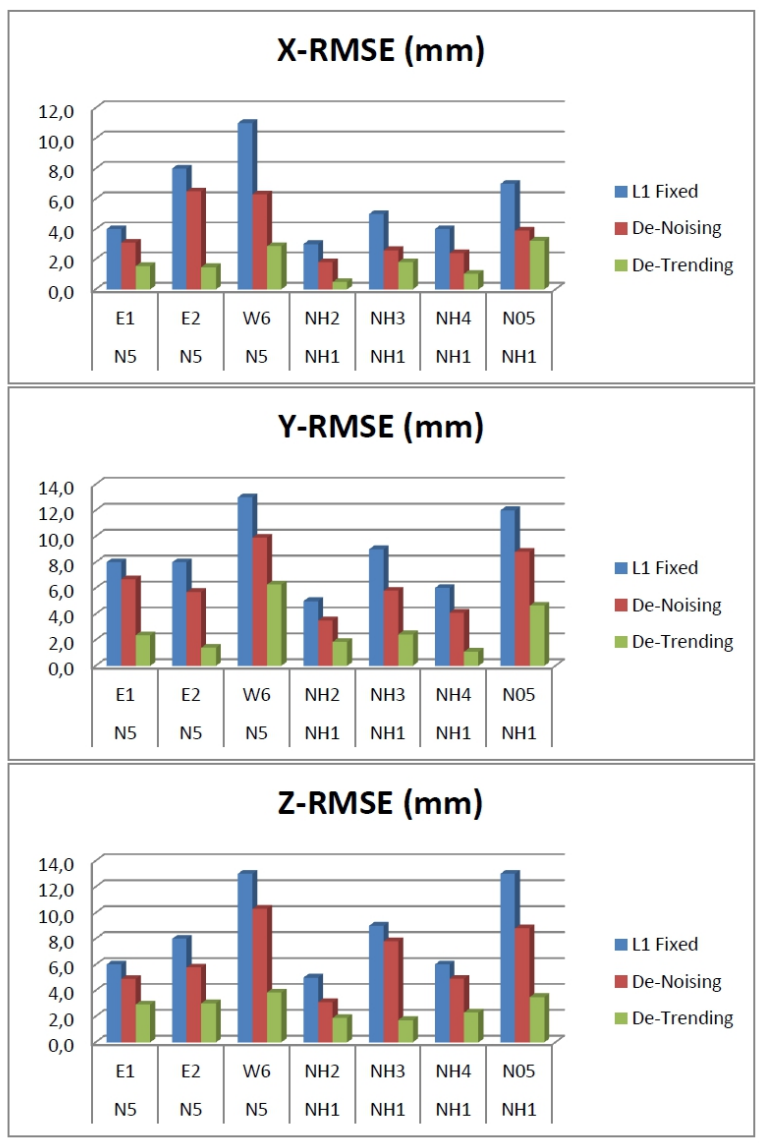

Figure 11. Coordinate RMS when computed with raw data (L1 Fixed), De-Noising and De-Trending techniques for short baselines.

\subsubsection{Long Baselines}

The second category baselines range from 20 to 50 kilometres. They are used to compute the coordinates for SBNK, AIR2, PRDS, $\mathrm{COCH}$ and STRA. Figure 12 and Figure 13 show the coordinate difference (bias) and the root mean square error of these coordinates with respect to the reference coordinates using the Bernese software.

\section{VERSITA}

In this category L1 float and ionospheric free solutions are used. The results for the long baselines $L 1$ float solution match the reference coordinates within 19-50 mm and RMS within $50 \mathrm{~mm}$. Wavelets Multi-Resolution is applied to the L1 measurement using Bior 3.3 with both the median threshold estimator and the detrending techniques to the eight levels of decomposition. The estimated error is used to correct each double difference measurement before the estimation process begins again. In addition, the performance of the wavelets de-trending technique is compared with the traditional wavelets de-noising using a median estimator. Figure 12 and Figure 13 show the bias and RMS reduction in the final solution ( $X, Y$ and $Z$ components) after correcting the double difference measurements using both de-trending and de-noising techniques. It can be seen from Figure 12 that the de-noising technique reduced the bias for long baselines from $15 \%$ at Y-Bias for NCAL-COCH baseline (from $18 \mathrm{~mm}$ to $15 \mathrm{~mm}$ ) to $42 \%$ at X-Bias for NCAL-COCH baseline (from $15 \mathrm{~mm}$ to $8.7 \mathrm{~mm}$ ). The RMS reductions for long baselines (Figure 13) vary between $25 \%$ at Z-RMS from baseline N5-PRDS (from $81 \mathrm{~mm}$ to $60 \mathrm{~mm}$ ) to $49 \%$ at Y-RMS for baseline N5-PRDS (from $58 \mathrm{~mm}$ to $29.8 \mathrm{~mm}$ ). The average bias reduction for all the $X, Y$ and $Z$ components is $30 \%$, while the average $\mathrm{RMS}$ reduction is $36 \%$ when using the de-noising technique.

The de-trending technique reduction to bias and RMS can be seen in the same figures. The bias for short baselines was reduced from $2 \%$ at Z-Bias for NCAL-AIR2 baseline (from $33 \mathrm{~mm}$ to $32.2 \mathrm{~mm}$ ) to $25 \%$ at X-Bias for NCAL-COCH baseline (from $15 \mathrm{~mm}$ to $11 \mathrm{~mm}$ ).

The RMS improvements for the long baseline L1 float solution vary from $56 \%$ at X-RMS from baseline NCAL-SBNK (from $32 \mathrm{~mm}$ to $14 \mathrm{~mm}$ ) to $89 \%$ at Z-RMS for baseline NCAL-COCH (from $45 \mathrm{~mm}$ to $5 \mathrm{~mm}$ ). The average bias reduction for all the $X, Y$ and $Z$ components is $14 \%$, while the average RMS reduction when using the de-noising technique is $75 \%$.

Figure 14 and Figure 15 show the bias and RMS reduction in the final solution ( $\mathrm{X}, \mathrm{Y}$ and $\mathrm{Z}$ Cartesian components) after correcting the double difference measurements using both de-trending and de-noising techniques. It can be seen from Figure 14 that the denoising technique reduced the bias for long baselines from $17.5 \%$ at Y-Bias for NCAL-COCH baseline (from $10 \mathrm{~mm}$ to $8.3 \mathrm{~mm}$ ) to $60 \%$ at X-Bias for N05-PRDS baseline (from $5 \mathrm{~mm}$ to $2 \mathrm{~mm}$ ). The RMS reductions for long baselines (Figure 15) vary between $22 \%$ at ZRMS from baseline NH1-PRDS (from $63 \mathrm{~mm}$ to $48 \mathrm{~mm}$ ) to $42 \%$ at 


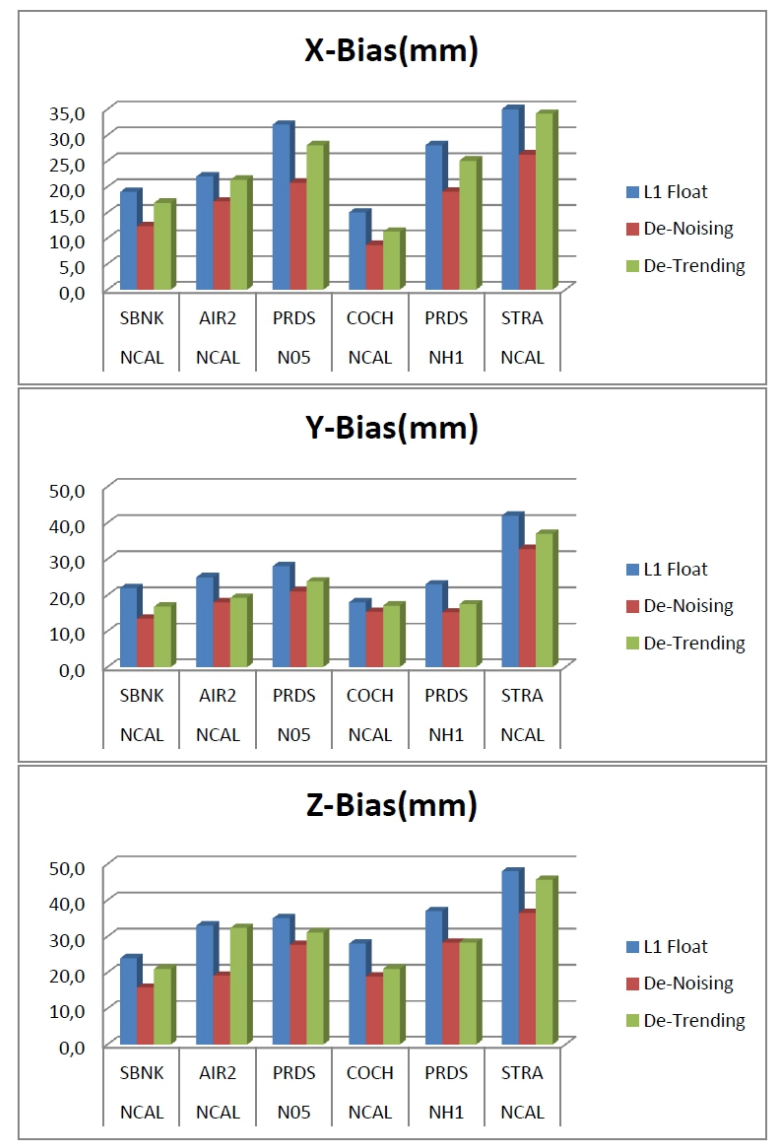

Figure 12. Coordinate bias when computed with raw data ( $L 1$ float), De-Noising and De-Trending techniques for long baselines.

X-RMS for baseline NCAL-STRA (from $21 \mathrm{~mm}$ to $12.2 \mathrm{~mm}$ ). The average bias reduction for all the $X, Y$ and $Z$ components is $20 \%$, while the average RMS reduction when using the de-noising technique is $33 \%$. The de-trending technique reduction to bias and RMS can be seen in the same figures. The bias for the long baselines was reduced from $3 \%$ at Y-Bias for NCAL-AIR2 baseline (from $3 \mathrm{~mm}$ to $2.9 \mathrm{~mm}$ ) to $35 \%$ at X-Bias for NCAL-COCH baseline (from $8 \mathrm{~mm}$ to $5.2 \mathrm{~mm}$ ). The RMS reductions, for the long baseline IF solution, vary between $54 \%$ at Z-RMS from baseline NCAL-AIR2 (from $18 \mathrm{~mm}$ to $8.3 \mathrm{~mm}$ ) to $86 \%$ at X-RMS for baseline NH1-PRDS (from $11 \mathrm{~mm}$ to $1.5 \mathrm{~mm}$ ). The average bias reduction for all the $X, Y$ and $Z$ components is $15 \%$, while the average RMS reduction when using the de-trending technique is $73 \%$.

\subsection{Conclusion}

Table 15 shows the summary of the analysis made in the previous sections. It can be seen from the table that the de-noising technique gives consistent results for both short and long baselines. The average bias reduction that can be achieved from the de-

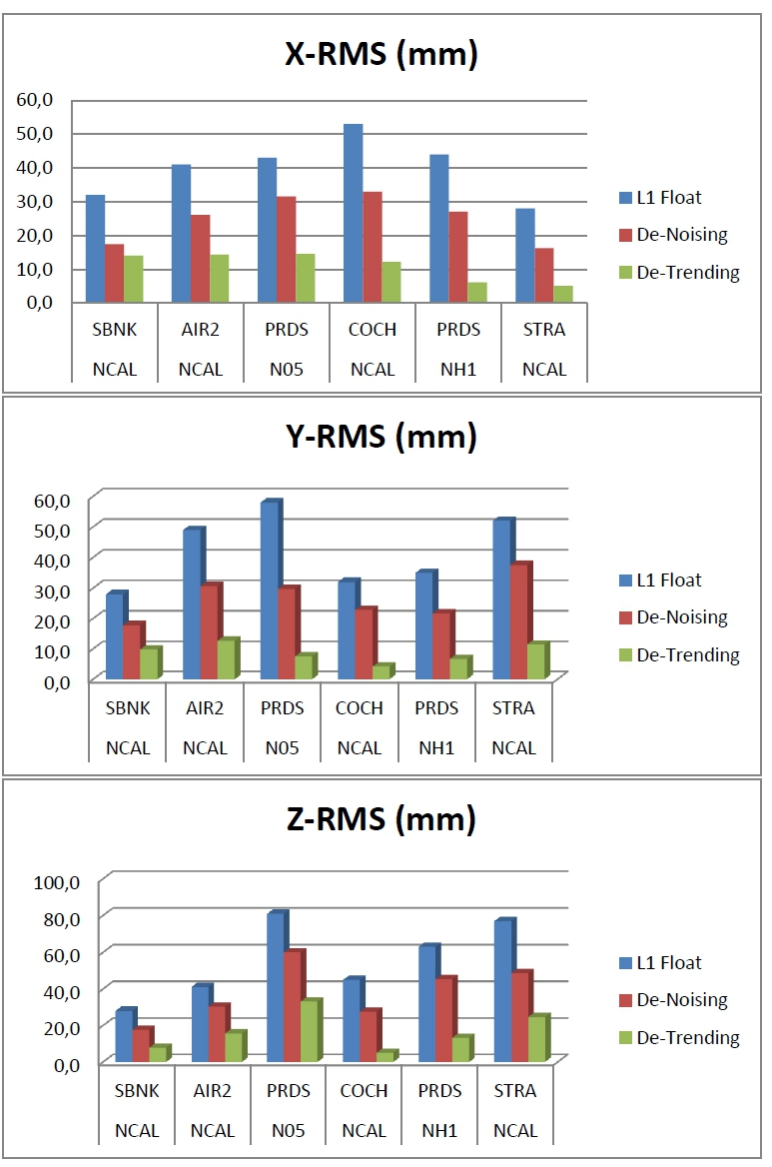

Figure 13. Coordinate RMS when computed with raw data (L1 Float), De-Noising and De-Trending techniques for long baselines.

noising technique is around $20-30 \%$ and the average RMS reduction is around $30-40 \%$. Moreover, the de-trending technique outperforms the de-noising technique for RMS improvement in short and long baselines. The performance in the de-trending technique is almost three times better than the traditional de-noising technique for bias and RMS reduction.

Although the de-trending technique out-performs the de-noising technique in the RMS reduction, it does produce inconsistent results for the bias reduction. The de-trending methodology performed impressively for short baselines in RMS and bias reduction as the average RMS and bias reduction were around $80 \%$. However, for longer baselines the bias reduction is minimal although the RMS reduction is still in the range of $70-80 \%$. It can be concluded that the de-trending technique can reduce the double difference errors dramatically for short baselines. Conversely, the de-trending technique can cause a biased solution for long baselines depending on the low frequency part of the error (ionosphere, low multipath), as it will enhance the RMS value and indicate good statistics for the solution but not enhance the bias to the same level. There-

\section{VERSITA}




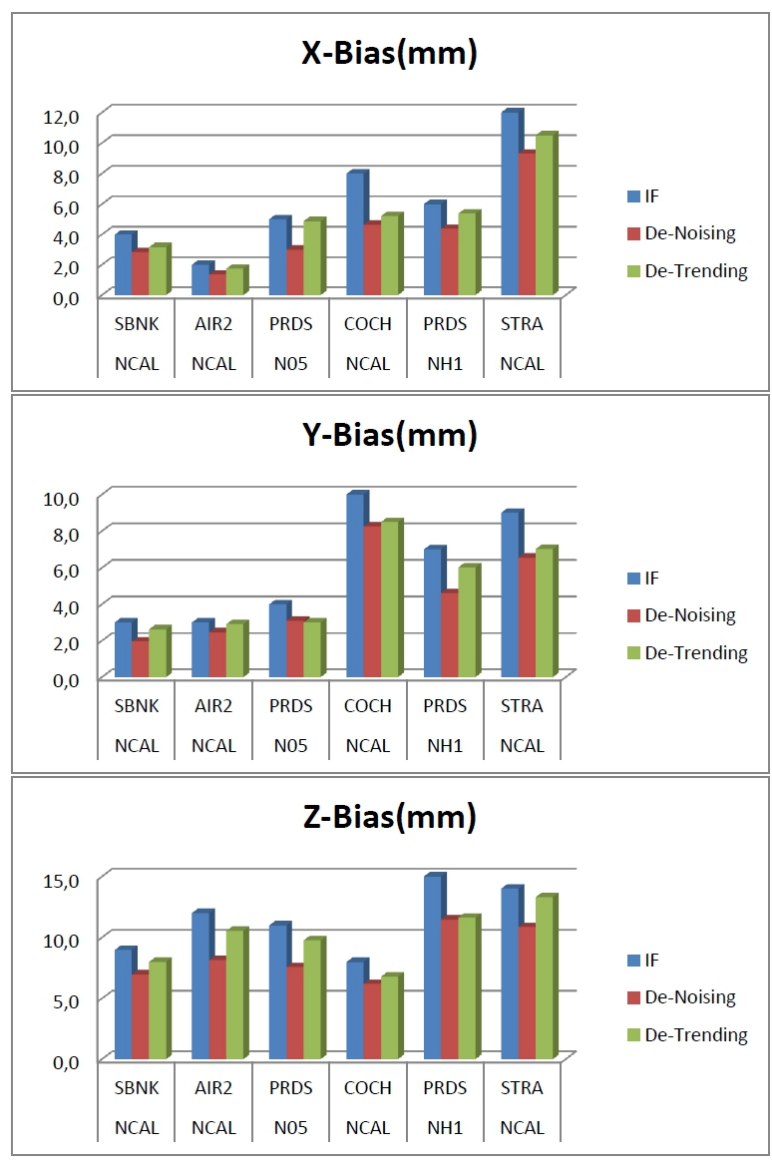

Figure 14. Coordinate bias when computed with raw data (IF), DeNoising and De-Trending techniques for long baselines.

Table 15. average bias and RMS reduction in percentage for fixed short baseline solution and long baseline float and IF solution.

\begin{tabular}{ccc}
\hline Fixed L1 & De-Noising & De-Trending \\
\hline Average Bias & 23.0 & 73.3 \\
\hline Average RMS & 29.4 & 68.5 \\
\hline Float L1 & De-Noising & De-Trending \\
\hline Average Bias & 29.5 & 13.7 \\
\hline Average RMS & 35.4 & 74.1 \\
\hline Float IF & De-Noising & De-Trending \\
\hline Average Bias & 28.9 & 14.6 \\
\hline Average RMS & 32.7 & 72.8 \\
\hline
\end{tabular}

fore, it is important to isolate ionospheric error by modeling (and not spectrum filtering) before dealing with multipath, as it is hard to separate between both errors in the spectral domain.

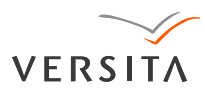

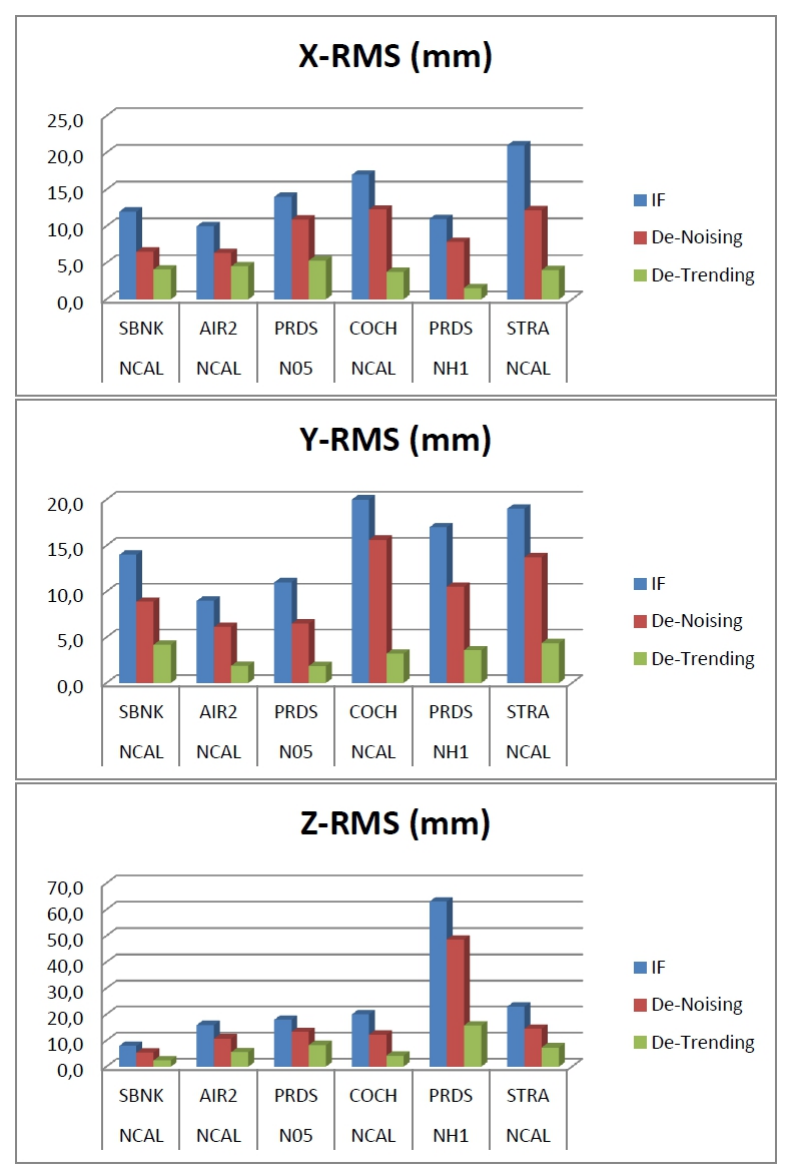

Figure 15. Coordinate RMS when computed with raw data (IF), DeNoising and De-Trending techniques for long baselines.

\section{Acknowledgments}

This study was supported in part by research funding from the Natural Science and Engineering Research Council of Canada (NSERC),Geomatics for Informed Decisions (GEOIDE), Network Centers of Excellence (NCE), of Dr. Naser El-Sheimy.

\section{References}

Dammalage T. L., Satirapod C., Kibe S. and Ogaja C., 2010, Wavelet transform application to C/A code multipath mitigation at GPS reference stations for improved differential GPS corrections, J. Sur. Rev., 42, 317, 240-255(16).

Donoho D. L. and Johnstone I. M., 1994, Ideal spatial adaptation by wavelet shrinkage, Biom., 81 425-55.

El-Ghazouly A., Elhabiby M. and El-Sheimy N., 2008, Wavelet- 
Based Carrier Phase Multipath Reduction, the United States and Trade Mark Office(Submitted,September 28th,2008.12 pages).

El-Ghazouly A., Elhabiby M. and El-Sheimy N., 2009, The use of Wavelets in GPS Error Analysis with Emphasis to Singularity Detection and Multipath Removal, Amer. Geoph. Union Joint Ass.

Elhabiby, M., 2007, Wavelet Representation of Geodetic Operators, Ph.D. Uni. of Calgary.

Han S. and Rizos C., 1997, Multipath effects on GPS in mine environments, 10th Int. Cong. of the Int. Soci. for Mine Sur., Fremantle, Australia, 447-457.

Hofmann-Wellenhof B., Lichtenegger H. and Collins J., 2007, Global Positioning System theory and practice. Springer Wien New York.

Hubbard B. B., 1998, The world according to wavelets: the story of a mathematical technique in the making. Wellesley, Mass.

Hugentobler U., Schaer S., Fridez P., Beutler G., Bock H., Brockmann E., Dach R., Gurtner W., Ineichen D., Johnson J., Meindl M., Mervart L., Springer T. and R. Weber (2001), Bernese GPS Software, Version 4.2, University of Bern, 2001.

Lee Y. W., Suh Y. C. and Shibasaki R., 2008, A simulation system for GNSS multipath mitigation using spatial statistical methods, Comp. \& Geos., 34(11), 1597-609.

Linlin Ge., Han S. and Rizos C., 2000, Multipath mitigation of continuous GPS measurements using an adaptive filter, GPS Sol., 4(2), 19-30.

Ogaja C. and Satirapod C., 2007, Analysis of high-frequency multipath in 1-Hz GPS kinematic solutions, GPS Sol., 11(4), 269-80.

Ogden R. T., 1997, Essential wavelets for statistical applications and data analysis, Birkhäuser.

Raquet J. and Lachapelle G., 1996, Determination and reduction of GPS reference station multipath using multiple receivers, $9^{\text {th }}$ Int. Tech. Meeting of the Sat. Div. of the Inst. of Nav., ION GPS-96. Part 1 (of 2), Kansas City, MO, USA, 673-81.

Ray J. K., 2000, Mitigation of GPS Code and Carrier Phase Multipath Effects using a Multi-Antenna System, Ph.D. thesis Univ. of Calgary.
Satirapod C. and Rizos C., 2005, Multipath mitigation by wavelet analysis for GPS base station applications, Sur. Rev., 38(295), 2-10.

Souza E. M. and Monico J. F. G., 2004, Wavelet shrinkage: High-frequency multipath reduction from GPS relative positioning, GPS Sol., 8(3), 152-9.

Tait M., Sheng L. and Cannon M. E., 2006, The Feasibility of Replacing Precise Levelling with GPS for Permafrost Deformation Monitoring, INGEO 2004 and FIG Reg. Cen. and Eastern Eur. Conf. on Eng. Surveying.

Teunissen P. J. G., 1993, Least squares estimation of the integer GPS ambiguities, General Meeting of the IAG.

Zhang Y. and Bartone C., 2004, Multipath mitigation in the frequency domain, PLANS, Piscataway, NJ, USA: IEEE, 486-95. 